\title{
Synthesis and Characterization of 2-phenyl-5- (1-phenyl-3-(3, 4, 5-trimethoxyphenyl)-1H-pyrazol- 4-yl) - 1, 3, 4-oxadiazole Scaffolds for Assessing Their Medicinal Potentials
}

\author{
Krishna Prasad Chennapragada',2, Venkata Sathya Machiraju Palagummi3,* \\ 'Department of Chemistry, Jawaharlal Nehru Technological University, Kakinada, Andhra Pradesh, INDIA. \\ Escientia Bio Pharma Pvt Ltd, ICICI Knowledge Park, Hyderabad, Telangana, INDIA. \\ ${ }^{3}$ Department of Chemistry, Pragati Engineering College (A), Surampalem, Andhra Pradesh, INDIA.
}

\begin{abstract}
Objective: The present research is aimed at the discovery and development of 1-phenyl-3(3,4,5-trimethoxyphenyl)-1 H-pyrazole derivatives as a series of novel 1, 3, 4-oxadiazoles (7a-7h) through iodine-catalyzed oxidative cyclization of the hydrazone derivatives (6a$6 \mathrm{~h}$ ) in the presence of potassium carbonate as base and DMSO as solvent in good toexcellent yields. Methods: The structures of all the newly synthesized compounds $16 a-$ $6 \mathrm{~h}$ ) and (7a-7h) were well characterized by IR, 1H NMR, 13C NMR and HRMS. Furthermore, allthe synthesized compounds (6a-6h and 7a-7h) were evaluated for their antimicrobial andanti-oxidant activities. Results: The research results revealed that the compound 6d (Z)-2-bromo-N'-((1-phenyl-3-(3, 4, 5-trimethoxyphenyl)-1H-pyrazol-4-yl) methylene) benzohydrazide) has antimicrobial potent while the synthesized compounds $6 \mathrm{a}(Z)-N$ '((1-phenyl-3-(3, 4, 5-trimethoxyphenyl)-1H-pyrazol-4-yl) methylene) benzohydrazide), $6 e$ (Z)-2, 5-dichloro-N'-((1-phenyl-3-(3, 4, 5-trimethoxyphenyl)-1H-pyrazol-4-yl) methylene) benzohydrazide), 7e (2-(2, 5-dichlorohenyl)-5-(1-phenyl-3-(3, 4, 5-trimethoxyphenyl)1H-pyrazol-4-yl)-1, 3, 4-oxadiazole) and 7f (2-(3, 5-dichlorophenyl)-5-(1-phenyl-3-(3, 4, 5-trimethoxyphenyl)-1H-pyrazol-4-yl)-1, 3, 4-oxadiazole) exhibited strong antioxidant activity compared to the control BHT. Conclusion: The novel hydrazonessynthesized and $1,3,4$ oxadiazole derivatives may be suggested for their establishment in chemical class of antimicrobial and antioxidant agents in new drug discovery and medicinal research.
\end{abstract}

Key words: Hydrazones, Oxadiazoles, Pyrazole, Anti-microbial, Anti-oxidant activity.

\section{INTRODUCTION}

The synthesis and biological evaluation of 1, 3, 4-oxadiazoles containing compounds have increased considerably in the last two decades. 1,3,4-Oxadiazole is an important heterocyclic compound which contains two carbons, two nitrogen's, one oxygen atom and two double bonds in a five-membered ring with general formula $\mathrm{C}_{2} \mathrm{H}_{2} \mathrm{ON}_{2}$. Potential pharmacological activity of 1,3 , 4-oxadiazoles is due to the presence of toxophoric $-\mathrm{N}=\mathrm{C}-\mathrm{O}$ linkage which may react with the nucleophilic centres of the microbial cell. 1, 3, 4-Oxadiazole and its derivatives has attracted considerable attention in the medicinal, material and pes- ticide chemistry. ${ }^{1}$ The various biological and pharmaceutical properties possessed by this important structural motif has attracted the researchers for the development of new drug molecules. ${ }^{2}$ The 1, 3, 4-oxadiazole compounds are generally synthesized by the oxidative cyclization of hydrazones which were prepared by the reaction of aromatic hydrazides with aromatic aldehydes. The various reagents employed for the oxidative cyclization of 1, 3, 4-Oxadiazole includes Lead tetraacetate, Lead dioxide, Potassium Permanganate, Chloramine-T, Ferric chloride and Iodobenzenediacetate. Examples of com-
Submission Date: 16-07-2017; Revision Date: 25-09-2017; Accepted Date: 10-01-2018

DOI: 10.5530/ijper.52.1.15 Correspondence:

Dr. P.V.S. Machiraju, Dean R \& D, Professor Of Chemistry, Pragathi Engineering College, Surampalem-533437, Near Kakinada, E.G.Dt, Andhra Pradesh, INDIA.

Phone no: +91-9246691641 Email: drpvsm.res@gmail. com

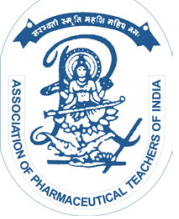

www.ijper.org 
pounds containing the 1, 3, 4-oxadiazole unit which are currently used in clinical medicine are: Raltegravir an antiretroviral drug and Zibotentan an anticancer agent, Vadrin a leprostatic drug and eudromil a hypnotic drug are a few examples.

1, 3, 4-oxadiazole derivatives have displayed a broad spectrum of biological activities such as antimicrobial ${ }^{3}$, antioxidant ${ }^{4}$, anticancer ${ }^{5}$, anticonvulsant ${ }^{6}$, antitubercular ${ }^{7}$, antiviral $^{8}$, antimalarial ${ }^{9}$ and analgesic and anti-inflammatory..$^{10}$ In addition to the various biological activities the 1,3,4-oxadiazoles are also found to possess potential agrochemical use ${ }^{11}$ due to their wide spectrum of insecticidal ${ }^{12}$ and acaricidal. ${ }^{13}$ Properties attained by interfering with insect's chitin biosynthesis. ${ }^{14}$ This class of materials also has gained the significance of modern applications such as fluorescence, scintillators and photographic materials. ${ }^{14}$ They are of great interest as structural units in polymeric membranes designed for gas separation applications. ${ }^{15}$ and as emitting layers in electroluminescent devices. ${ }^{16}$ respectively.

Among the 1, 3, 4-oxadiazoles, pyrazole containing 2, 5-disubstituted-1, 3, 4-oxadiazoles are found to have considerable interest in medicinal chemistry because of their various biological characterized possessed by these compounds like antimicrobial, ${ }^{17}$ antioxidant, ${ }^{18}$ DNA photocleavage activity, ${ }^{19}$ anticancer, ${ }^{20}$ anti-inflammatory ${ }^{21}$ and antivira ${ }^{22}$ activities.

On the other hand, hydrazones are of considerable interest in synthetic chemistry and are broadly considered for studies as reaction intermediates or reactants since they can readily undergo various ring closure reactions. ${ }^{23}$ Hydrazones are molecules containing highly reactive azomethine group (CO-NH-N=CH) and thus useful in new drug development. ${ }^{24}$ The hydrazones can easily be synthesised by the condensation of an aldehyde or ketone with primary amine. ${ }^{25}$ Hydrazones were also found adequate importance in organic synthesis since they served as an intermediate compounds in the synthesis of heterocyclic compounds like 1, 3, 4-oxadiazoles. ${ }^{26}$ The hydrazone derivatives are found to posses various biological activities such as antimicrobial ${ }^{27}$, antioxidant ${ }^{28}$, antitubercular, ${ }^{29}$ anti cancer, ${ }^{30}$ antiproliferative, ${ }^{31}$ analgesic and anti-inflammatory ${ }^{32}$ respectively. Among the hydrazone derivatives pyrazole substituted hydrazone derivatives are known to possess various biological activities such as antibacterial,,$^{33}$ antifungal, ${ }^{34}$ anticancer, ${ }^{35}$ antioxidant, ${ }^{36}$ anti-inflammatory, ${ }^{37}$ antitumor and antiangiogenesis ${ }^{38}$ and antiplatelet activities. ${ }^{39}$

Pyrazole framework plays significant role in biologically active compounds and represents an interesting template for medicinal and combinatorial chemistry. ${ }^{40}$ Compounds containing this heterocycle exhibit antimicrobial, ${ }^{41}$ anti-oxidant, ${ }^{42}$ anti-inflammatory, ${ }^{43}$ antitubercular, ${ }^{44}$ anticancer activity, ${ }^{45}$ antiviral, ${ }^{46}$ antidepressant and anticonvulsant activities. ${ }^{47}$ Some arylpyrazoles were reported to have non-nucleoside HIV-1 reverse transcriptase inhibitory activity. ${ }^{48}$ Extensive studies have been dedicated to arylpyrazole derivatives such as Celecoxib, a well-known cyclooxygenase- 2 inhibitor. ${ }^{49}$

Based on the above-mentioned literary observations and with a view to search for new, potent, selective, and less toxic antimicrobial and anti-oxidant agents it is proposed to synthesize some novel pyrazole-linked hydrazones which were further converted into 2 , 5-disubstituted 1, 3, 4-oxadiazoles.

\section{MATERIALS AND METHODS}

The chemicals employed for the present research work were commercially procured from Alfa Aesar. Melting points were determined in open glass capillaries on a Stuart SMP30 apparatus and are uncorrected. IR spectra were recorded as KBr pellets on a Shimadzu FTIR $8400 \mathrm{~S}$ spectrophotometer. ${ }^{1} \mathrm{H}$ NMR $(400 \mathrm{MHz})$ and ${ }^{13} \mathrm{C}$ NMR $(100 \mathrm{MHz})$ spectra were recorded on a Bruker DPX 400 spectrophotometer using tetramethylsilane (TMS) as an internal standard, $\mathrm{CDCl}_{3}$ and DMSO- $d 6$ as solvents and the signals are reported as s (singlet), d (doublet), dd (doublet of doublet), $\mathrm{t}$ (triplet), q (quartet), $\mathrm{m}$ (multiplet) and coupling constants in Hz. HRMS spectra were recorded on a Xevo QTof mass spectrometer. Silica gel

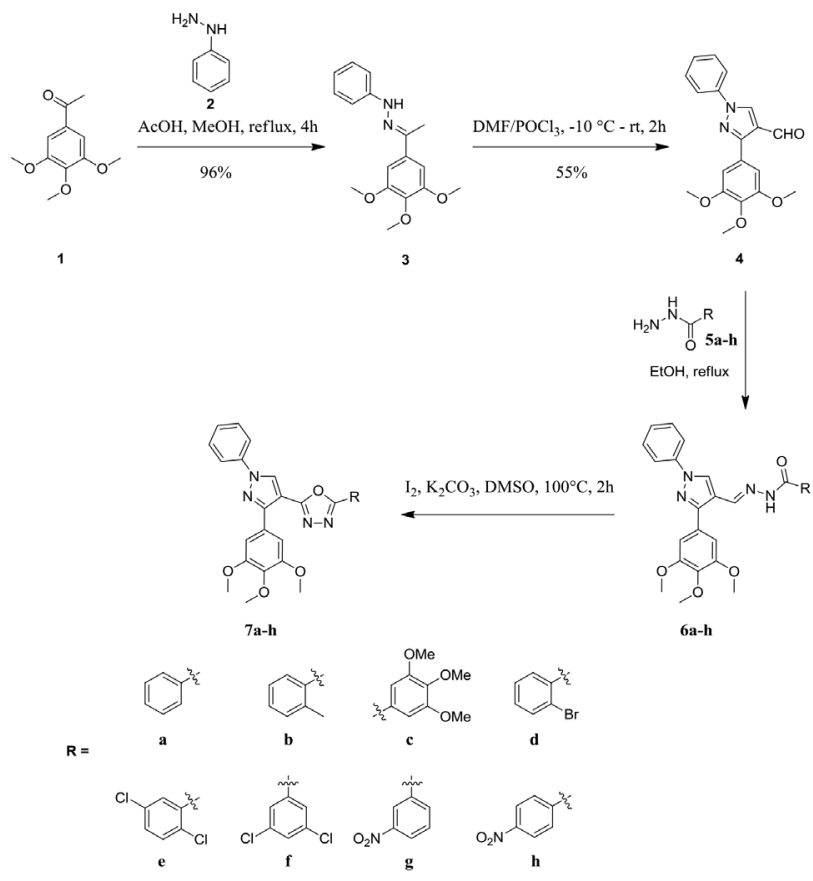

Scheme 1: Synthesis of 2-phenyl-5-(1-phenyl-3-(3, 4, 5-trimethoxyphenyl)-1H-pyrazol-4-yl)-1, 3, 4-oxadiazoles (7a-7h). 
60 F24 of Merck pre-coated plates were employed for their thin layer chromatography (TLC) analysis to check the purity of the compounds, the spot being located under UV light and iodine vapours.

\section{Synthesis}

The title compounds were synthesized by the following steps.

Synthesis of 1-(1-(3, 4, 5-trimethoxyphenyl) ethylidene)-2-phenylhydrazine (3)..$^{50}$

To a solution of 1-(3, 4, 5-trimethoxyphenyl) ethanone $1(5 \mathrm{~g}, 19 \mathrm{mmol})$ in methanol $(40 \mathrm{~mL})$ was added phenyl hydrazine 2 (2.1 g, $19 \mathrm{mmol})$ followed by glacial acetic acid $(15 \mathrm{~mL})$ at room temperature. The resulting reaction mixture was heated to reflux for $3 \mathrm{~h}$. TLC showed completion of starting material (TLC System: $20 \%$ Ethylacetate in hexane, $\left.R_{f}: 0.5\right)$. The solvent was evaporated under reduced pressure, diluted with ice water $(25 \mathrm{~mL})$, stirred for $15 \mathrm{~min}$ at $10^{\circ} \mathrm{C}$ and filtered the precipitated solid, washed with ice water $(20 \mathrm{~mL})$ followed by n-pentane $(20 \mathrm{~mL})$ and dried under vacuum to afford 1-(1-(3, 4, 5-trimethoxyphenyl) ethylidene)-2-phenylhydrazine (3).

Synthesis of 3-(3, 4, 5-trimethoxypheyl)-1-phenyl1H-pyrazole-4 carbaldehyde (4). ${ }^{51}$

Phosphorus oxychloride $(6.2 \mathrm{ml}, 66 \mathrm{mmol})$ was added to $\mathrm{N}, \mathrm{N}$-Dimethylformamide $(26.5 \mathrm{ml}, 339 \mathrm{mmol})$ at -5 to $0^{\circ} \mathrm{C}$ drop wise over a period of $10 \mathrm{~min}$, stirred at the same temperature for $30 \mathrm{~min}$ and added compound $\mathbf{3}$ (6.6 g, $22 \mathrm{mmol})$ dissolved in DMF $(10 \mathrm{ml})$ dropwise at $-5^{\circ} \mathrm{C}$ over a period of $15 \mathrm{~min}$. The reaction mixture was allowed to warm to room temperature and stirred for $1.5 \mathrm{~h}$. The completion of the reaction was monitored by TLC. After completion, the reaction mixture was slowly added to ice cold water and basified with saturated $\mathrm{NaHCO}_{3}$ solution to adjust the $\mathrm{pH} 7-8$, stirred for $1 \mathrm{~h}$ at room temperature and filtered the precipitated solid, washed with water and dried under vacuum to get the crude compound. The crude compound was washed with methanol, filtered and dried under vacuum to afford pure compound 4 .

\section{General procedure for the synthesis of hydrazone derivatives $6 a-6 h^{52}$}

A suspension of 1-phenyl-3-(3, 4, 5-trimethoxyphenyl)1H-pyrazole-4-carbaldehyde $4(0.44 \mathrm{mmol})$ and hydrazides $(\mathbf{5 a}-\mathbf{5 h})(0.44 \mathrm{mmol})$ in ethanol $(7 \mathrm{~mL})$ was heated to reflux for 7-8 h. After completion, the precipitated solid was filtered, washed with minimum amount of ethanol and dried under vacuum to obtain hydrazone derivatives (6a-6h).

\section{General procedure for the synthesis of 1, 3,}

\section{4-oxadiazole dervatives $7 a-7 h .{ }^{53}$}

To a stirred solution of compound (6a-6h) $(0.22 \mathrm{mmol})$ in DMSO was added anhydrous $\mathrm{K}_{2} \mathrm{CO}_{3}(0.66 \mathrm{mmol})$ followed by Iodine $(0.26 \mathrm{mmol})$ and heated to $100^{\circ} \mathrm{C}$ for $1 \mathrm{~h}$. After completion of reaction, the reaction mixture was cooled to room temperature and treated with $5 \% \mathrm{Na}_{2} \mathrm{~S}_{2} \mathrm{O}_{3}$ solution $(5 \mathrm{ml})$, extracted with ethylacetate $(25 \mathrm{ml})$. The organic layer was separated and washed with water and brine solution, dried over anhydrous $\mathrm{Na}_{2} \mathrm{SO}_{4}$ concentrated under reduced pressure to obtain the crude product. The crude solid product was washed with diethyl ether, filtered and dried under vacuum to afford oxadiazole derivatives (7a-7h).

\section{The physicochemical and the spectral data of the synthesized compounds (6a-6h) and (7a-7h) are depicted as follows}

(Z)-N'-((1-phenyl-3-(3, 4, 5-trimethoxyphenyl)1H-pyrazol-4-yl) methylene) benzohydrazide (6a). Off-white solid, M.P: $213-215^{\circ} \mathrm{C}$. Yield: $75 \%$. Chemical formula: $\mathrm{C}_{26} \mathrm{H}_{24} \mathrm{~N}_{4} \mathrm{O}_{4}$. FT-IR $\left(\mathrm{KBr}, \mathrm{cm}^{-1}\right): 1649(\mathrm{C}=\mathrm{O})$, $1557(\mathrm{C}=\mathrm{N}), 1125$ (Ar-O). ${ }^{1} \mathrm{H}$ NMR $(400 \mathrm{MHz}, \mathrm{DMSO}-$ $\left.d_{6}, \delta \mathrm{ppm}\right): 3.71\left(* 3.72, \mathrm{~s}, 3 \mathrm{H}, \mathrm{OCH}_{3}\right), 3.87(* 3.82, \mathrm{~s}, 6 \mathrm{H}$, $\left.2 \mathrm{OCH}_{3}\right), 6.99(* 7.08, \mathrm{~s}, 2 \mathrm{H}, \mathrm{Ar}-\mathrm{H}), 7.36$ (t, 1H, Ar-H, $\mathrm{J}=7.2 \mathrm{~Hz}), 7.48-7.56(\mathrm{~m}, 5 \mathrm{H}, \mathrm{Ar}-\mathrm{H}), 7.87(\mathrm{~d}, 2 \mathrm{H}$, Ar-H, J = 7.6 Hz), 8.01 (d, 2H, Ar-H, J = 8.0 Hz), 8.57 $(* 8.67, \mathrm{~s}, 1 \mathrm{H}, \mathrm{CH}=\mathrm{N}), 8.97$ (*9.10, s, $1 \mathrm{H}$, Pyrazole-H), 11.67 (br s,1H, NH). ${ }^{13} \mathrm{C}$ NMR $(100 \mathrm{MHz}$, DMSO-d 6 , $\delta \mathrm{ppm})$ : 56.02, 60.06, 106.02, 116.91, 118.89, 126.96, 127.44, 127.54, 128.43, 129.57, 131.62, 133.57, 137.88, 139.03, 141.16, 151.97, 152.99, 162.77. HRMS: Calculated for $\mathrm{C}_{26} \mathrm{H}_{24} \mathrm{~N}_{4} \mathrm{O}_{4}, 456.18$, found: $457.1705[\mathrm{M}+\mathrm{H}]^{+}$ (Z)-2-methyl-N'-((1-phenyl-3-(3，4，5-trimethoxyphenyl)-1H-pyrazol-4-yl) methylene) benzohydrazide (6b). Off-white solid, M.P: $206-208^{\circ} \mathrm{C}$. Yield: $71 \%$. Chemical formula: $\mathrm{C}_{27} \mathrm{H}_{26} \mathrm{~N}_{4} \mathrm{O}_{4}$. FT-IR $\left(\mathrm{KBr}, \mathrm{cm}^{-1}\right): 1651(\mathrm{C}=\mathrm{O}), 1555(\mathrm{C}=\mathrm{N}), 1127(\mathrm{Ar}-\mathrm{O})$. ${ }^{1} \mathrm{H}$ NMR (400 MHz, DMSO- $d_{6}, \delta$ ppm): 2.48 (s, 3H, $\left.\mathrm{Ar}-\mathrm{CH}_{3}\right), 3.70$ (s, $\left.3 \mathrm{H}, \mathrm{OCH}_{3}\right), 3.86\left(\mathrm{~s}, 6 \mathrm{H}, 2 \mathrm{OCH}_{3}\right), 6.98$ (s, 2H, Ar-H), 7.25-7.29 (m, 2H, Ar-H), 7.30-7.42 (m, 3H, Ar-H), 7.53 (t, 2H, Ar-H, J = $7.76 \mathrm{~Hz}), 8.02(\mathrm{~d}, 2 \mathrm{H}$, Ar-H, J = 8.0 Hz), 8.44 (s, 1H, CH=N), 8.98 (s, 1H, Pyrazole-H), 11.59 (br s, $1 \mathrm{H}, \mathrm{NH}) \cdot{ }^{13} \mathrm{C}$ NMR $(100$ $\mathrm{MHz}, \mathrm{DMSO}-d 6, \delta$ ppm): 19.24, 56.00, 60.07, 105.89, $116.84,118.86,125.64,126.97,127.36,127.42,127.50$, $129.60,129.83,130.58,135.45,135.78,137.86,139.03$, 140.78, 151.81, 152.99, 164.82. HRMS: Calculated for $\mathrm{C}_{27} \mathrm{H}_{26} \mathrm{~N}_{4} \mathrm{O}_{4}, 470.20$, found: $471.1907[\mathrm{M}+\mathrm{H}]^{+}$

(Z)-3, 4, 5-trimethoxy-N'-((1-phenyl-3-(3, 4, 5-trimethoxyphenyl)-1H-pyrazol-4-yl) methylene) benzohydrazide (6c): Off-white solid, M.P: 203-205 ${ }^{\circ}$ C. Yield: $82 \%$. Chemical formula: $\mathrm{C}_{29} \mathrm{H}_{30} \mathrm{~N}_{4} \mathrm{O}_{7}$. 
FT-IR $\left(\mathrm{KBr}, \mathrm{cm}^{-1}\right): 1636(\mathrm{C}=\mathrm{O}), 1583(\mathrm{C}=\mathrm{N}), 1123$ (Ar-O). ${ }^{1} \mathrm{H}$ NMR (400 MHz, DMSO- $d, \delta$ ppm): 3.71 (s, $\left.6 \mathrm{H}, 2 \mathrm{OCH}_{3}\right), 3.84\left(\mathrm{~s}, 6 \mathrm{H}, 2 \mathrm{OCH}_{3}\right), 3.87\left(\mathrm{~s}, 6 \mathrm{H}, 2 \mathrm{OCH}_{3}\right)$, 7.01 (s, 2H, Ar-H), 7.18 (s, 2H, Ar-H), 7.38 (t, 1H, Ar-H, $\mathrm{J}=7.2 \mathrm{~Hz}), 7.53(\mathrm{t}, 2 \mathrm{H}, \mathrm{Ar}-\mathrm{H}, \mathrm{J}=7.68 \mathrm{~Hz}), 8.02(\mathrm{~d}, 2 \mathrm{H}$, Ar- $\mathrm{H}, \mathrm{J}=8.08 \mathrm{~Hz}), 8.55(\mathrm{~s}, 1 \mathrm{H}, \mathrm{CH}=\mathrm{N}), 8.99(\mathrm{~s}, 1 \mathrm{H}$, Pyrazole-H), 11.53 (br s, $1 \mathrm{H}, \mathrm{NH}) .{ }^{13} \mathrm{C}$ NMR $(100 \mathrm{MHz}$, DMSO- $d 6, \delta$ ppm): 56.05, 56.13, 60.08, 60.13, 105.25, 106.10, 116.89, 118.91, 126.99, 127.46, 127.53, 128.79, 129.59, 137.94, 139.03, 140.39, 141.14, 151.95, 152.69, 153.01, 162.37. HRMS: Calculated for $\mathrm{C}_{29} \mathrm{H}_{30} \mathrm{~N}_{4} \mathrm{O}_{7}$, 546.21, found: $547.1993[\mathrm{M}+\mathrm{H}]$

(Z)-2-bromo-N'-((1-phenyl-3-(3, 4, 5-trimethoxyphenyl)-1H-pyrazol-4-yl) methylene) benzohydrazide (6d). Off-white solid, M.P: $177-179^{\circ} \mathrm{C}$. Yield: $70 \%$. Chemical formula: $\mathrm{C}_{26} \mathrm{H}_{23} \mathrm{BrN}_{4} \mathrm{O}_{4}$. FT-IR $\left(\mathrm{KBr}, \mathrm{cm}^{-1}\right)$ : $1648(\mathrm{C}=\mathrm{O}), 1551(\mathrm{C}=\mathrm{N}), 1127$ (Ar-O). ${ }^{1} \mathrm{H}$ NMR $(400$ $\left.\mathrm{MHz}, \mathrm{DMSO}-d_{6}, \delta \mathrm{ppm}\right): 3.70\left(* 3.72, \mathrm{~s}, 3 \mathrm{H}, \mathrm{OCH}_{3}\right)$, $3.86\left(* 3.84, \mathrm{~s}, 6 \mathrm{H}, 2 \mathrm{OCH}_{3}\right), 6.99(* 6.83, \mathrm{~s}, 2 \mathrm{H}, \mathrm{Ar}-\mathrm{H})$, 7.35-7.41 (m, 2H, Ar-H), 7.42-7.56 (m, 4H, Ar-H), 7.72 (*7.63, d, 1H, Ar-H, J = 7.84), 8.02 (*7.83, d, 2H, Ar-H, $\mathrm{J}=8.08), 8.41(* 8.30, \mathrm{~s}, 1 \mathrm{H}, \mathrm{CH}=\mathrm{N}), 9.0(\mathrm{~s}, 1 \mathrm{H}$, Pyrazole-H), $11.72(* 11.81$, br s, $1 \mathrm{H}, \mathrm{NH}) .{ }^{13} \mathrm{C}$ NMR $(100$ $\mathrm{MHz}$, DMSO- $d 6, \delta$ ppm): 56.01, 60.07, 105.65, 105.89, $116.53,116.61,118.87,119.07,119.46,126.26,127.00$, $127.18,127.35,127.74,129.04,129.26,129.59,130.61$, 131.46, 132.16, 132.83, 137.47, 137.64, 137.88, 138.89, 139.00, 141.45, 151.72, 151.87, 152.99, 162.91, 168.66. HRMS: Calculated for $\mathrm{C}_{26} \mathrm{H}_{23} \mathrm{BrN}_{4} \mathrm{O}_{4}, 534.09$, found: $535.0853[\mathrm{M}+\mathrm{H}]^{+}$

(Z)-2, 5-dichloro-N'-((1-phenyl-3-(3, 4, 5-trimethoxyphenyl)-1H-pyrazol-4-yl) methylene) benzohydrazide (6e). Off-white solid, M.P: $179-181^{\circ} \mathrm{C}$. Yield: $76 \%$. Chemical formula: $\mathrm{C}_{26} \mathrm{H}_{22} \mathrm{Cl}_{2} \mathrm{~N}_{4} \mathrm{O}_{4}$. FT-IR $\left(\mathrm{KBr}, \mathrm{cm}^{-1}\right): 1654(\mathrm{C}=\mathrm{O}), 1561(\mathrm{C}=\mathrm{N}), 1127(\mathrm{Ar}-\mathrm{O})$. ${ }^{1} \mathrm{H}$ NMR (400 MHz, DMSO- $\left.d_{6}, \delta \mathrm{ppm}\right): 3.70$ (*3.71, s, $\left.3 \mathrm{H}, \mathrm{OCH}_{3}\right), 3.86\left(* 3.83, \mathrm{~s}, 6 \mathrm{H}, 2 \mathrm{OCH}_{3}\right), 6.99(* 6.81$, s, $2 \mathrm{H}, \mathrm{Ar}-\mathrm{H}), 7.35-7.39$ (m, 1H, Ar-H), 7.50-7.59 (m, 4H, Ar-H), 7.71 (s, 1H, Ar-H), 8.02 (*7.86, d, 2H, Ar-H, $\mathrm{J}=7.92 \mathrm{~Hz}), 8.40(* 8.23, \mathrm{~s}, 1 \mathrm{H}, \mathrm{CH}=\mathrm{N}), 9.01(\mathrm{~s}, 1 \mathrm{H}$, Pyrazole-H), $11.80(* 11.93$, br s, $1 \mathrm{H}, \mathrm{NH}) .{ }^{13} \mathrm{C}$ NMR (100 MHz, DMSO-d6, $\delta$ ppm): 56.02, 60.06, 105.66, 105.94, 116.47, 118.89, 119.09, 126.58, 127.03, 127.30, $127.83,128.42,128.66,128.95,129.31,129.59,130.34$, $130.79,131.14,131.53,131.87,136.75,137.90,138.45$, 138.97, 141.94, 151.77, 151.96, 152.98, 160.57, 166.51. HRMS: Calculated for $\mathrm{C}_{26} \mathrm{H}_{22} \mathrm{Cl}_{2} \mathrm{~N}_{4} \mathrm{O}_{4}, 524.10$, found: $525.0982[\mathrm{M}+\mathrm{H}]^{+}$

(Z)-3, 5-dichloro-N'-((1-phenyl-3-(3, 4, 5-trimethoxyphenyl)-1H-pyrazol-4-yl) methylene) benzohydrazide (6f). Off-white solid, M.P: $224-226^{\circ} \mathrm{C}$. Yield: $84 \%$. Chemical formula: $\mathrm{C}_{26} \mathrm{H}_{22} \mathrm{Cl}_{2} \mathrm{~N}_{4} \mathrm{O}_{4}$. FT-IR
$\left(\mathrm{KBr}, \mathrm{cm}^{-1}\right): 1686(\mathrm{C}=\mathrm{O}), 1555(\mathrm{C}=\mathrm{N}), 1120(\mathrm{Ar}-\mathrm{O})$. ${ }^{1} \mathrm{H}$ NMR (400 MHz, DMSO- $d, \delta$ ppm): 3.72 (s, $3 \mathrm{H}$, $\left.\mathrm{OCH}_{3}\right), 3.88\left(\mathrm{~s}, 6 \mathrm{H}, 2 \mathrm{OCH}_{3}\right), 6.99$ (s, 2H, Ar-H), 7.38 $(\mathrm{t}, 1 \mathrm{H}, \mathrm{Ar}-\mathrm{H}, \mathrm{J}=7.4 \mathrm{~Hz}), 7.54(\mathrm{t}, 2 \mathrm{H}, \mathrm{Ar}-\mathrm{H}, \mathrm{J}=7.76$ $\mathrm{Hz})$, 7.86-7.91 (m, 3H, Ar- $\mathrm{H}), 8.02$ (d, 2H, Ar-H, $\mathrm{J}=7.96 \mathrm{~Hz}), 8.54(\mathrm{~s}, 1 \mathrm{H}, \mathrm{CH}=\mathrm{N}), 9.01(\mathrm{~s}, 1 \mathrm{H}$, Pyrazole$\mathrm{H}), 11.80$ (s, $1 \mathrm{H}, \mathrm{NH}) .{ }^{13} \mathrm{C}$ NMR $(100 \mathrm{MHz}$, DMSO-d6, $\delta \mathrm{ppm}): 56.06,60.07,106.10,116.64,118.94,126.34$, 127.04, 127.35, 127.65, 129.58, 131.01, 134.39, 136.78, 137.96, 138.99, 142.24, 152.16, 153.00, 159.97. HRMS: Calculated for $\mathrm{C}_{26} \mathrm{H}_{22} \mathrm{Cl}_{2} \mathrm{~N}_{4} \mathrm{O}_{4}, 524.10$, found: 525.1005 $[\mathrm{M}+\mathrm{H}]^{+}$

(Z)-3-nitro-N'-((1-phenyl-3-(3, 4, 5-trimethoxyphenyl)-1H-pyrazol-4-yl) methylene) benzohydrazide (6g): Pale yellow solid, M.P: $224-226^{\circ} \mathrm{C}$. Yield: $76 \%$. Chemical formula: $\mathrm{C}_{26} \mathrm{H}_{23} \mathrm{~N}_{5} \mathrm{O}_{6}$. FT-IR $\left(\mathrm{KBr}, \mathrm{cm}^{-1}\right): 1689(\mathrm{C}=\mathrm{O}), 1559(\mathrm{CH}=\mathrm{N}), 1125(\mathrm{Ar}-\mathrm{O})$. ${ }^{1} \mathrm{H}$ NMR (400 MHz, DMSO- $\left.d_{6}, \delta \mathrm{ppm}\right): 3.72$ (s, 3H, $\left.\mathrm{OCH}_{3}\right), 3.88\left(\mathrm{~s}, 6 \mathrm{H}, 2 \mathrm{OCH}_{3}\right), 7.01(\mathrm{~s}, 2 \mathrm{H}, \mathrm{Ar}-\mathrm{H}), 7.37$ (t, 1H, Ar- $\mathrm{H}, \mathrm{J}=7.24 \mathrm{~Hz}$ ), 7.54 (t, 2H, Ar-H, J = 7.8 $\mathrm{Hz}), 7.83$ (t, 1H, Ar-H, J = 7.96 Hz), 8.03 (d, 2H, Ar-H, $\mathrm{J}=8.0 \mathrm{~Hz}$ ), 8.34 (d, 1H, Ar-H, J = $7.92 \mathrm{~Hz}), 8.43$ $(\mathrm{d}, 1 \mathrm{H}, \mathrm{Ar}-\mathrm{H}, \mathrm{J}=8.16 \mathrm{~Hz}), 8.59(\mathrm{~s}, 1 \mathrm{H}, \mathrm{CH}=\mathrm{N}), 8.72$ (s, 1H, Ar-H), 9.02 (s, 1H, Pyrazole-H), 11.96 (s, 1H, NH). ${ }^{13} \mathrm{C}$ NMR (100 MHz, DMSO- $d 6, \delta$ ppm): 56.06, 60.07, 106.13, 116.68, 118.94, 122.23, 126.24, 127.03, 127.37, 127.66, 129.58, 130.29, 134.01, 134.92, 137.96, 138.99, 142.26, 147.79, 152.16, 153.01, 160.58. HRMS: Calculated for $\mathrm{C}_{26} \mathrm{H}_{23} \mathrm{~N}_{5} \mathrm{O}_{6}$ 501.16, found: $502.1605[\mathrm{M}+\mathrm{H}]^{+}$ (Z)-4-nitro-N'-((1-phenyl-3-(3, 4, 5-trimethoxyphenyl)-1H-pyrazol-4-yl) methylene) benzohydrazide (6h). Pale yellow solid, M.P: $243-245^{\circ} \mathrm{C}$. Yield: $74 \%$. Chemical formula: $\mathrm{C}_{26} \mathrm{H}_{23} \mathrm{~N}_{5} \mathrm{O}_{6}$. FT-IR $\left(\mathrm{KBr}, \mathrm{cm}^{-1}\right): 1681(\mathrm{C}=\mathrm{O}), 1593(\mathrm{CH}=\mathrm{N}), 1120$ (Ar-O). ${ }^{1} \mathrm{H}$ NMR (400 MHz, DMSO- $\left.d_{6}, \delta \mathrm{ppm}\right): 3.73$ (s, 3H, $\left.\mathrm{OCH}_{3}\right), 3.89\left(* 3.84, \mathrm{~s}, 6 \mathrm{H}, 2 \mathrm{OCH}_{3}\right), 7.01(* 6.88, \mathrm{~s}$, $2 \mathrm{H}, \mathrm{Ar}-\mathrm{H}), 7.39$ (t, $1 \mathrm{H}, \mathrm{Ar}-\mathrm{H}, \mathrm{J}=7.2 \mathrm{~Hz}), 7.55(\mathrm{t}, 2 \mathrm{H}$, Ar-H, J = $7.6 \mathrm{~Hz}$ ), 8.04 (*7.93, d, 3H, Ar-H, J = 8.0 $\mathrm{Hz}), 8.14(* 8.29, \mathrm{~d}, 2 \mathrm{H}, \mathrm{Ar}-\mathrm{H}, \mathrm{J}=8.8 \mathrm{~Hz}), 8.37$ (d, 2H, Ar-H, J $=8.8 \mathrm{~Hz}), 8.60(* 8.70, \mathrm{~s}, 1 \mathrm{H}, \mathrm{CH}=\mathrm{N}), 9.03$ (s, 1H, Pyrazole-H), 11.96 (br s, $1 \mathrm{H}, \mathrm{NH}) \cdot{ }^{13} \mathrm{C}$ NMR (100 MHz, DMSO-d6, $\delta$ ppm): 56.07, 60.09, 106.11, $116.68,118.96,123.65,127.06,127.37,127.69,129.10$, 129.60, 137.97, 139.01, 139.24, 142.39, 149.22, 152.17, 153.02, 161.09. HRMS: Calculated for $\mathrm{C}_{26} \mathrm{H}_{23} \mathrm{~N}_{5} \mathrm{O}_{6}$, 501.16, found: $502.1582[\mathrm{M}+\mathrm{H}]^{+}$

2-phenyl-5-(1-phenyl-3-(3, 4, 5-trimethoxyphenyl)1H-pyrazol-4-yl)-1, 3, 4-oxadiazole (7a): Off- white solid, M.P: $199-201^{\circ} \mathrm{C}$. Yield: $73 \%$. Chemical formula: $\mathrm{C}_{26} \mathrm{H}_{22} \mathrm{~N}_{4} \mathrm{O}_{4}$. FT-IR $\left(\mathrm{KBr}, \mathrm{cm}^{-1}\right): 1588(\mathrm{C}=\mathrm{N}), 1124$ (ArO). ${ }^{1} \mathrm{H}$ NMR (400 MHz, $\left.\mathrm{CDCl}_{3}, \delta \mathrm{ppm}\right): 3.92$ (s, 6H, $\left.2 \mathrm{OCH}_{3}\right), 3.93\left(\mathrm{~s}, 3 \mathrm{H}, \mathrm{OCH}_{3}\right), 7.32$ (s, 2H, Ar-H), 7.38- 
7.41 (m, 1H, Ar- H), 7.47-7.55 (m, 5H, Ar-H), 7.83-7.85 (m, 2H, Ar-H), 7.99-8.01 (m, 2H, Ar-H), 8.66 (s, 1H, Pyrazole-H). ${ }^{13} \mathrm{C}$ NMR (100 MHz, DMSO- $d 6, \delta$ ppm): 55.99, 60.12, 105.83, 106.38, 119.02, 123.27, 126.45, $126.78,127.48,129.37,129.68,131.46,131.99,138.24$, 138.66, 150.38, 152.64, 159.60, 163.09. HRMS: Calculated for $\mathrm{C}_{26} \mathrm{H}_{22} \mathrm{~N}_{4} \mathrm{O}_{4}$, 454.16, found: $455.1639[\mathrm{M}+\mathrm{H}]^{+}$ 2-(1-phenyl-3-(3, 4, 5-trimethoxyphenyl)-1H-pyrazol-4-yl)-5-(o-tolyl)-1, 3, 4-oxadiazole (7b): Offwhite solid, M.P: $204-206^{\circ} \mathrm{C}$. Yield: $90 \%$. Chemical formula: $\mathrm{C}_{27} \mathrm{H}_{24} \mathrm{~N}_{4} \mathrm{O}_{4}$. FT-IR $\left(\mathrm{KBr}, \mathrm{cm}^{-1}\right): 1584(\mathrm{C}=\mathrm{N})$, 1120 (Ar-O). ${ }^{1} \mathrm{H}$ NMR (400 MHz, DMSO- $d, \delta$ ppm): 2.61(s, 3H, Ar- $\left.\mathrm{CH}_{3}\right), 3.73$ (s, 3H, $\left.\mathrm{OCH}_{3}\right), 3.81$ (s, 6H, $\left.2 \mathrm{OCH}_{3}\right), 7.35-7.46(\mathrm{~m}, 5 \mathrm{H}, \mathrm{Ar}-\mathrm{H}), 7.48-7.52(\mathrm{~m}, 1 \mathrm{H}$, Ar-H), 7.59 (t, 2H, Ar-H, J = $7.72 \mathrm{~Hz}), 7.94$ (d, 1H, Ar-H, J =7.72 Hz), 8.05 (d, 2H, Ar-H, J =7.92 Hz), 9.43 (s, 1H, Pyrazole-H). ${ }^{13} \mathrm{C}$ NMR (100 MHz, DMSO-d6, $\delta \mathrm{ppm}): \quad 21.47,56.01,60.12,105.89,106.48,119.09$, $122.37,126.33,126.87,127.48,128.71,129.68,131.30$, 131.43, 131.75, 137.67, 138.24, 138.69, 150.55, 152.65, 159.11, 163.42. HRMS: Calculated for $\mathrm{C}_{27} \mathrm{H}_{24} \mathrm{~N}_{4} \mathrm{O}_{4}$, 468.18, found: $469.1665[\mathrm{M}+\mathrm{H}]^{+}$

2-(1-phenyl-3-(3, 4, 5-trimethoxyphenyl)-1H-pyrazol-4-yl)-5-(3, 4, 5-trimethoxyphenyl)-1, 3, 4-oxadiazole (7c): Off-white solid, M.P: $177-179^{\circ} \mathrm{C}$. Yield: $83 \%$. Chemical formula: $\mathrm{C}_{29} \mathrm{H}_{28} \mathrm{~N}_{4} \mathrm{O}_{7}$. FT-IR ( $\mathrm{KBr}$, $\left.\mathrm{cm}^{-1}\right): 1588(\mathrm{C}=\mathrm{N}), 1122$ (Ar-O). ${ }^{1} \mathrm{H}$ NMR $(400 \mathrm{MHz}$, $\left.\mathrm{CDCl}_{3}, \delta \mathrm{ppm}\right): 3.91\left(\mathrm{~s}, 18 \mathrm{H}, 6 \mathrm{OCH}_{3}\right), 7.18(\mathrm{~s}, 2 \mathrm{H}$, Ar-H), 7.24 (d, 2H, Ar-H, J = $12.36 \mathrm{~Hz}), 7.40$ (t, 1H, Ar-H, J = 7.2 Hz), 7.54 (t, 2H, Ar-H, J = 7.64 Hz), 7.85 (d, $2 \mathrm{H}, \mathrm{Ar}-\mathrm{H}, \mathrm{J}=7.96 \mathrm{~Hz}), 8.69$ (s, 1H, Pyrazole-H). ${ }^{13} \mathrm{C}$ NMR (100 MHz, DMSO-d6, $\delta$ ppm): 55.98, 56.09, $60.09,60.24,103.84,105.86,106.40,118.42,119.11$, 126.87, 127.50, 129.68, 131.24, 138.17, 138.66, 140.58, 150.46, 152.59, 153.47, 159.49, 162.95. HRMS: Calculated for $\mathrm{C}_{29} \mathrm{H}_{28} \mathrm{~N}_{4} \mathrm{O}_{7}, 544.20$, found: $545.1902[\mathrm{M}+\mathrm{H}]^{+}$ 2-(2-bromophenyl)-5-(1-phenyl-3-(3, 4, 5-trimethoxyphenyl)-1H-pyrazol-4-yl)-1, 3, 4-oxadiazole (7d): Off-white solid, M.P: $209-211^{\circ} \mathrm{C}$. Yield: $73 \%$. Chemical formula: $\mathrm{C}_{26} \mathrm{H}_{21} \mathrm{BrN}_{4} \mathrm{O}_{4}$. FT-IR $\left(\mathrm{KBr}, \mathrm{cm}^{-1}\right): 1586(\mathrm{C}=\mathrm{N})$, 1123 (Ar-O). ${ }^{1} \mathrm{H}$ NMR (400 MHz, $\left.\mathrm{CDCl}_{3}, \delta \mathrm{ppm}\right): 3.91$ $\left(\mathrm{s}, 3 \mathrm{H}, \mathrm{OCH}_{3}\right), 3.93\left(\mathrm{~s}, 6 \mathrm{H}, 2 \mathrm{OCH}_{3}\right), 7.35-7.49(\mathrm{~m}, 5 \mathrm{H}$, Ar-H), 7.54 (t, 2H, Ar-H, J = 7.6 Hz), 7.75 (d, 1H, Ar-H, $\mathrm{J}=7.92 \mathrm{~Hz}), 7.84(\mathrm{~d}, 2 \mathrm{H}, \mathrm{Ar}-\mathrm{H}, \mathrm{J}=7.76 \mathrm{~Hz}), 7.92-7.94$ (dd, $1 \mathrm{H}$, Ar- H, J = 1.52, $7.68 \mathrm{~Hz}$ ), 8.64 (s, 1H, PyrazoleH). ${ }^{13} \mathrm{C}$ NMR $(100 \mathrm{MHz}$, DMSO- $d 6, \delta \mathrm{ppm}): 56.00$, 60.11, 105.56, 106.45, 119.15, 119.29, 120.85, 124.52, 126.69, 127.54, 128.17, 128.51, 129.68, 131.48, 131.61, 133.28, 134.39, 138.26, 138.64, 150.59, 152.66, 159.93, 162.03. HRMS: Calculated for $\mathrm{C}_{26} \mathrm{H}_{21} \mathrm{BrN}_{4} \mathrm{O}_{4}, 532.07$, found: $533.0682[\mathrm{M}+\mathrm{H}]^{+}$
2-(2,5 dichlorophenyl)-5-(1-phenyl-3-(3, 4, 5-trimethoxyphenyl)-1H-pyrazol-4-yl)-1, 3, 4-oxadiazole (7e): Off-white solid, M.P: $219-221^{\circ} \mathrm{C}$. Yield: $79 \%$. Chemical formula: $\mathrm{C}_{26} \mathrm{H}_{20} \mathrm{Cl}_{2} \mathrm{~N}_{4} \mathrm{O}_{4}$ FT-IR $\left(\mathrm{KBr}, \mathrm{cm}^{-1}\right)$ : $1586(\mathrm{C}=\mathrm{N}), 1120(\mathrm{Ar}-\mathrm{O}) .{ }^{1} \mathrm{H}$ NMR $\left(400 \mathrm{MHz}, \mathrm{CDCl}_{3}\right.$, $\delta \mathrm{ppm}): 3.92\left(\mathrm{~s}, 3 \mathrm{H}, \mathrm{OCH}_{3}\right), 3.93\left(\mathrm{~s}, 6 \mathrm{H}, 2 \mathrm{OCH}_{3}\right), 7.32$ (s, 2H, Ar-H), 7.39-7.44 (m, 2H, Ar-H), $7.48(\mathrm{~d}, 1 \mathrm{H}$, Ar-H, J = 8.6 Hz), 7.54 (t, 2H, Ar-H, J = 7.6 Hz), 7.84 (d, $2 \mathrm{H}, \mathrm{Ar}-\mathrm{H}, \mathrm{J}=7.72 \mathrm{~Hz}$ ), 8.01 (d, 1H, Ar-H, J = 2.4 $\mathrm{Hz}), 8.65$ (s, 1H, Pyrazole-H). ${ }^{13} \mathrm{C}$ NMR $(100 \mathrm{MHz}$, DMSO- $d 6, \delta$ ppm): 56.01, 60.10, 105.49, 106.50, 119.17, $123.88,126.71,127.58,129.69,130.21,130.55,131.65$, 132.29, 132.69, 133.03, 138.27, 138.63, 150.66, 152.65, 160.02, 160.17. HRMS: Calculated for $\mathrm{C}_{26} \mathrm{H}_{20} \mathrm{Cl}_{2} \mathrm{~N}_{4} \mathrm{O}_{4}$, 522.09, found: $523.0849[\mathrm{M}+\mathrm{H}]^{+}$

2-(3, 5-dichlorophenyl)-5-(1-phenyl-3-(3, 4, 5-trimethoxyphenyl)-1H-pyrazol-4-yl)-1, 3, 4-oxadiazole (7f): Off-white solid, M.P: $181-183^{\circ} \mathrm{C}$. Yield: $81 \%$. Chemical formula: $\mathrm{C}_{26} \mathrm{H}_{20} \mathrm{Cl}_{2} \mathrm{~N}_{4} \mathrm{O}_{4}$ FT-IR $(\mathrm{KBr}$, $\left.\mathrm{cm}^{-1}\right): 1589(\mathrm{C}=\mathrm{N}), 1129(\mathrm{Ar}-\mathrm{O}) .{ }^{1} \mathrm{H} \mathrm{NMR}(400 \mathrm{MHz}$, DMSO- $\left.d_{6}, \delta \mathrm{ppm}\right): 3.74\left(\mathrm{~s}, 3 \mathrm{H}, \mathrm{OCH}_{3}\right), 3.84(\mathrm{~s}, 6 \mathrm{H}$, $\left.2 \mathrm{OCH}_{3}\right), 7.42-7.45(\mathrm{~m}, 3 \mathrm{H}, \mathrm{Ar}-\mathrm{H}), 7.60$ (t, 2H, Ar- $\mathrm{H}$, $\mathrm{J}=7.72 \mathrm{~Hz}), 7.92(\mathrm{t}, 1 \mathrm{H}, \mathrm{Ar}-\mathrm{H}, \mathrm{J}=1.8 \mathrm{~Hz}), 8.02-8.05$ (m, 4H, Ar-H), 9.52 (s, $1 \mathrm{H}$, Pyrazole-H). ${ }^{13} \mathrm{C}$ NMR (100 $\mathrm{MHz}$, DMSO- $d 6, \delta$ ppm): 56.01, 60.13, 105.58, 106.46, $119.05,124.88,126.49,126.68,127.58,129.71,131.24$, 131.83, 135.24, 138.29, 138.59, 150.43, 152.64, 160.26, 160.91. HRMS: Calculated for $\mathrm{C}_{26} \mathrm{H}_{20} \mathrm{Cl}_{2} \mathrm{~N}_{4} \mathrm{O}_{4}, 522.09$, found: $523.0869[\mathrm{M}+\mathrm{H}]$

2-(3-nitrophenyl)-5-(1-phenyl-3-(3，4，5-trimethoxyphenyl)-1H-pyrazol-4-yl)-1, 3, 4-oxadiazole (7g): Pale yellow solid, M.P: $176-178^{\circ} \mathrm{C}$. Yield: $77 \%$. Chemical formula: $\mathrm{C}_{26} \mathrm{H}_{21} \mathrm{~N}_{5} \mathrm{O}_{6}$. FT-IR $\left(\mathrm{KBr}, \mathrm{cm}^{-1}\right)$ : $1590(\mathrm{C}=\mathrm{N}), 1514\left(\mathrm{Ar}-\mathrm{NO}_{2}\right), 1126$ (Ar-O). ${ }^{1} \mathrm{H}$ NMR (400 MHz, DMSO- $\left.d_{6}, \delta \mathrm{ppm}\right): 3.75\left(\mathrm{~s}, 3 \mathrm{H}, \mathrm{OCH}_{3}\right)$, $3.83\left(\mathrm{~s}, 6 \mathrm{H}, 2 \mathrm{OCH}_{3}\right), 7.42-7.45(\mathrm{~m}, 3 \mathrm{H}, \mathrm{Ar}-\mathrm{H}), 7.60$ (t, 2H, Ar-H, J = $7.64 \mathrm{~Hz}), 7.92$ (t, 1H, Ar-H, J = 8.04 $\mathrm{Hz}), 8.05$ (d, 2H, Ar-H, J = $7.84 \mathrm{~Hz}$ ), 8.47 (d, 2H, Ar-H, $\mathrm{J}=7.92 \mathrm{~Hz}$ ), 8.71 (s, 1H, Ar-H), 9.53 (s, 1H, Pyrazole-H). ${ }^{13} \mathrm{C}$ NMR (100 MHz, DMSO- $d 6, \delta$ ppm): 55.99, 60.10, 105.54, 106.40, 119.08, 120.85, 124.83, 126.32, 126.68, $127.55,129.69,131.28,131.76,132.45,138.29,138.61$, 148.28, 150.46, 152.65, 160.26, 161.59. HRMS: Calculated for $\mathrm{C}_{26} \mathrm{H}_{21} \mathrm{~N}_{5} \mathrm{O}_{6}$, 499.15, found: $500.1482[\mathrm{M}+\mathrm{H}]^{+}$ 2-(4-nitrophenyl)-5-(1-phenyl-3-(3, 4, 5-trimethoxyphenyl)-1H-pyrazol-4-yl)-1, 3, 4-oxadiazole (7h): Pale yellow solid, M.P: $219-221^{\circ} \mathrm{C}$. Yield: $70 \%$. Chemical formula: $\mathrm{C}_{26} \mathrm{H}_{21} \mathrm{~N}_{5} \mathrm{O}_{6}$. FT-IR $\left(\mathrm{KBr}, \mathrm{cm}^{-1}\right)$ : $1594(\mathrm{C}=\mathrm{N}), 1517\left(\mathrm{Ar}-\mathrm{NO}_{2}\right), 1120$ (Ar-O). ${ }^{1} \mathrm{H}$ NMR (400 MHz, DMSO- $\left.d_{6}, \delta \mathrm{ppm}\right): 3.76\left(\mathrm{~s}, 3 \mathrm{H}, \mathrm{OCH}_{3}\right)$, $3.84\left(\mathrm{~s}, 6 \mathrm{H}, 2 \mathrm{OCH}_{3}\right), 7.44-7.47(\mathrm{~m}, 3 \mathrm{H}, \mathrm{Ar}-\mathrm{H}), 7.61$ (t, 2H, Ar-H, J = 8.0 Hz), 8.06 (d, 2H, Ar-H, J = 8.0 Hz), 
$8.30(\mathrm{~d}, 2 \mathrm{H}, \mathrm{Ar}-\mathrm{H}, \mathrm{J}=8.8 \mathrm{~Hz}), 8.46$ (d, 2H, Ar-H, $\mathrm{J}=8.8 \mathrm{~Hz}$ ), 9.52 (s, 1H, Pyrazole-H). ${ }^{13} \mathrm{C}$ NMR (100 MHz, DMSO-d6, $\delta$ ppm): 56.07, 60.17, 105.58, 106.44, 119.09, 124.65, 126.66, 127.63, 127.77, 128.91, 129.77, 131.89, 138.35, 138.64, 149.21, 150.52, 152.69, 160.57, 161.83. HRMS: Calculated for $\mathrm{C}_{26} \mathrm{H}_{21} \mathrm{~N}_{5} \mathrm{O}_{6}$, 499.15, found: $500.1472[\mathrm{M}+\mathrm{H}]^{+}$

\section{ANTIMICROBIAL EVALUATION 54}

Antibacterial Activity Test Microorganisms and Growth Media Stapbylococcus aureus (MTCC 3160), Bacillus cereus (MTCC 1305), E.Coli (MTCC 443) and Pseudomonas aeruginosa (MTCC 2453) and Candida albicans were selected based on their clinical and pharmacological importance. The bacterial strains obtained from Department of Microbiology, Osmania University, were employed for evaluating antibacterial activity. The bacterial stock cultures were incubated for $24 \mathrm{~h}$ at $37^{\circ} \mathrm{C}$ on nutrient agar. The bacteria were grown on Mueller-Hinton agar plates at $37^{\circ} \mathrm{C}$. The stock culture was maintained at $4^{\circ} \mathrm{C}$. for the growth of fungi potato dextrose agar was used. Whatman No.1 filter paper discs of $5 \mathrm{~mm}$ diameter were autoclaved by keeping in a clean and dry Petri plate. The discs were soaked in compound solutions for $5 \mathrm{~h}$ were taken as test material. After $5 \mathrm{~h}$ the discs were shade dried. The concentrations of compound solutions per disc are accounted for $0.1 \mathrm{~g} / 1 \mathrm{ml}$. Subsequently, they were carefully transferred to spread on cultured Petri plates. Filter paper discs immersed in ethanol, hexane, benzene and distilled water are prepared and used as control. To test the antibacterial activity, LB agar medium was prepared and the medium was sterilized at $121^{\circ} \mathrm{C}$ for 30 mins. The agar plates were prepared by pouring about $10 \mathrm{ml}$ of the medium into $10 \mathrm{~cm}$ petri dishes under aseptic condition and left undisturbed for $2 \mathrm{~h}$ to solidify the medium. $1 \mathrm{ml}$ of inocculum (containing suspension) of Staphylococcus aureus, Bacillus subtilis, E.Coli and was poured on to the plates separately containing solidified agar media. The prepared sterile filter paper discs were impregnated with the compound solutions and shaken thoroughly and these test plates incubated for a period of $48 \mathrm{hrs}$ in $\mathrm{BOD}$ at $37^{\circ} \mathrm{C}$ for the development of inhibitory zones and the average of 2 independent readings for each organism in different compound solutions were recorded. The inhibition zones were measured after 1 day at $37^{\circ} \mathrm{C}$ for bacteria. The diameter of the inhibition zone was measured and recorded with the aid of plastic ruler. Five paper discs placed in one Petri plate.

\section{ANTIOXIDANT EVALUATION 55}

The DPPH radical-scavenging activity was determined by using the method proposed by Yen and Chen (1995). DPPH $(100 \mathrm{lM})$ was dissolved in pure ethanol $(96 \%)$. The radical stock solution was prepared fresh daily. The DPPH solution $(1 \mathrm{ml})$ was added to $1 \mathrm{ml}$ of polyphenol extracts with $3 \mathrm{ml}$ of ethanol. The mixture was shaken vigorously and allowed to stand at room temperature in the dark for $10 \mathrm{~min}$. The decrease in absorbance of the resulting solution was monitored at $517 \mathrm{~nm}$ at $10 \mathrm{~min}$. The results were corrected for dilution and expressed in $1 \mathrm{M}$ Trolox per $100 \mathrm{~g}$ dry weight (dw). All determinations were performed in triplicate. 2, 6-bis (1, 1-dimethylethyl)-4-methylphenol (BHT) antioxidant agents was used as positive control.

\section{RESULTS AND DISCUSSION}

\section{Chemistry}

The route for the synthesis of 2, 5-substituted 1, 3, 4-oxadiazole derivatives (7a-h) is depicted in Scheme 1 which involves four steps. Initially, the starting material 3,4,5-trimethoxy acetophenone 1 was treated with phenyl hydrazine 2 using acetic acid and methanol as a solvent at reflux temperature to obtain the acetophenone phenylhydrazone derivative 3 in $96 \%$ yield. In the second step the acetophenone phenylhydrazone derivative 3 was treated with Vilsmeier-Haack reagent (DMF-POCl $)$ to obtain 1-phenyl-3-(3, 4, 5-trimethoxyphenyl)- $1 H$-pyrazole carboxaldehyde 4 in $50 \%$ yield. The obtained pyrazole carboxaldehyde intermediate 4 was treated with different aryl hydrazides $(\mathbf{5 a}-\mathbf{h})$ in the presence of ethanol at reflux temperature to obtain the corresponding hydrazone derivatives $(\mathbf{6 a}-\mathbf{h})$ which underwent iodine catalysed cyclisation using potassium carbonate as base and DMSO as solvent to afford the title compounds (7a-h) in 70-90\% yield. All the compounds synthesized were well characterized by IR, ${ }^{1} \mathrm{H}$ NMR, ${ }^{13} \mathrm{C}$ NMR and HRMS spectral analysis. It is considerable to note that hydrazones derivatives were found to be present as a mixture of two rotameric forms in solution. ${ }^{56}$ e.g. antiperiplanar (ap) and synperiplanar $(s p)$ as indicated by their ${ }^{1} \mathrm{H}$ NMR spectra. The ${ }^{1} \mathrm{H}$ NMR spectra of the hydrazone compounds $\mathbf{6 a}, \mathbf{6 d}, \mathbf{6 e}$ and $\mathbf{6 h}$ revealed the duplication of some signals indicating the presence of rotamers.

\section{Antimicrobial activity}

The title compounds (6a-h) and (7a-h) were evaluated for their antimicrobial activity using Escherichia Coli (MTCC 443) and Pseudomonas aeruginosa (MTCC 2453) as $\mathrm{g}$ negative bacterial strains and Staphylococcus aureus (MTCC 3160), Bacillus cereus (MTCC 1305) as g posi- 
Table 1: Antimicrobial activity (zone of inhibition in $\mathrm{mm}$ ) of compounds $6 \mathrm{a}-\mathrm{h}$ at various concentrations $(200,300$ and $500 \mu \mathrm{g} / \mathrm{mL})$.

\begin{tabular}{|c|c|c|c|c|c|c|c|c|c|c|c|c|c|c|c|}
\hline \multirow{4}{*}{ Compound } & \multicolumn{12}{|c|}{ Antibacterial activity (conc., $\mu \mathrm{g} / \mathrm{mL}$ ) } & \multicolumn{3}{|c|}{ Antifungal activity } \\
\hline & \multicolumn{6}{|c|}{ G negative bacteria } & \multicolumn{6}{|c|}{ G positive bacteria } & & & \\
\hline & \multicolumn{3}{|c|}{$\begin{array}{c}\text { E.coli } \\
\text { (MTCC 443) }\end{array}$} & \multicolumn{3}{|c|}{$\begin{array}{l}\text { P. aeruginosa } \\
\text { (MTCC 2453) }\end{array}$} & \multicolumn{3}{|c|}{$\begin{array}{c}\text { S.aureus } \\
\text { (MTCC 3160) }\end{array}$} & \multicolumn{3}{|c|}{$\begin{array}{c}\text { B. cereus } \\
\text { (MTCC 1305) }\end{array}$} & \multicolumn{3}{|c|}{ C.albicans } \\
\hline & 200 & 300 & 500 & 200 & 300 & 500 & 200 & 300 & 500 & 200 & 300 & 500 & 200 & 300 & 500 \\
\hline $6 a$ & 6 & 8 & 10 & 4 & 5 & 8 & 1 & 2 & 3 & 3 & 5 & 7 & 3 & 5 & 7 \\
\hline $6 b$ & 4 & 6 & 8 & - & - & - & 8 & 9 & 12 & 3 & 5 & 7 & - & - & - \\
\hline $6 c$ & - & - & - & 5 & 7 & 9 & - & - & - & - & - & - & - & - & - \\
\hline $6 d$ & - & - & - & - & - & - & 9 & 11 & 12 & - & - & - & 6 & 7 & 8 \\
\hline $6 e$ & - & - & - & - & - & - & 6 & 7 & 8 & 1 & 2 & 3 & 5 & 6 & 7 \\
\hline $6 f$ & 4 & 6 & 8 & 3 & 4 & 5 & 8 & 8 & 12 & 2 & 4 & 5 & - & - & - \\
\hline $6 g$ & - & - & - & 5 & 7 & 8 & - & - & - & - & - & - & - & - & - \\
\hline $6 h$ & - & - & - & 5 & 8 & 9 & - & - & - & - & - & - & 1 & 2 & 3 \\
\hline Streptomycin & 8 & 9 & 11 & 7 & 9 & 10 & 9 & 10 & 13 & 7 & 8 & 9 & 9 & 9 & 10 \\
\hline
\end{tabular}

Table 2: Antimicrobial activity (zone of inhibition in $\mathrm{mm}$ ) of compounds $7 \mathrm{a}-\mathrm{h}$ at various concentrations (200, 300 and $500 \mu \mathrm{g} / \mathrm{mL}$ ).

\begin{tabular}{|c|c|c|c|c|c|c|c|c|c|c|c|c|c|c|c|}
\hline \multirow{4}{*}{ Compound } & \multicolumn{12}{|c|}{ Antibacterial activity } & \multicolumn{3}{|c|}{ Antifungal activity } \\
\hline & \multicolumn{6}{|c|}{ G negative bacteria } & \multicolumn{6}{|c|}{ G positive bacteria } & \multirow{2}{*}{\multicolumn{3}{|c|}{ C.albicans }} \\
\hline & \multicolumn{3}{|c|}{$\begin{array}{c}\text { E.coli } \\
\text { (MTCC 443) }\end{array}$} & \multicolumn{3}{|c|}{$\begin{array}{l}P . \text { aeruginosa } \\
\text { (MTCC 2453) }\end{array}$} & \multicolumn{3}{|c|}{$\begin{array}{c}\text { S.aureus } \\
\text { (MTCC 3160) }\end{array}$} & \multicolumn{3}{|c|}{$\begin{array}{l}\text { B. cereus } \\
\text { (MTCC 1305) }\end{array}$} & & & \\
\hline & 200 & 300 & 500 & 200 & 300 & 500 & 200 & 300 & 500 & 200 & 300 & 500 & 200 & 300 & 500 \\
\hline $7 a$ & - & - & - & - & - & - & - & - & - & - & - & - & - & - & - \\
\hline $7 \mathrm{~b}$ & - & - & - & - & - & - & - & - & - & - & - & - & - & - & - \\
\hline $7 c$ & - & - & - & - & - & - & - & - & - & - & - & - & - & - & - \\
\hline $7 d$ & - & - & - & 2 & 3 & 4 & - & - & - & - & - & - & - & - & - \\
\hline $7 e$ & 3 & 4 & 5 & - & - & - & 3 & 4 & 5 & 1 & 2 & 3 & 3 & 4 & 6 \\
\hline $7 f$ & - & - & - & - & - & - & - & - & - & 2 & 3 & 4 & - & - & - \\
\hline $7 g$ & - & - & - & - & - & - & - & - & - & - & - & - & - & - & - \\
\hline $7 \mathrm{~h}$ & - & - & - & - & - & - & - & - & - & - & - & - & - & - & - \\
\hline Streptomycin & 7 & 8 & 10 & 8 & 9 & 10 & 9 & 10 & 11 & 8 & 8 & 9 & 9 & 9 & 10 \\
\hline
\end{tabular}

tive bacterial strains at different concentration levels of 200, 300 and $500 \mu \mathrm{g}$ with Streptomycin as standard drug. The related antibacterial activities of the hydrazone derivatives are present in Table 1. The compounds 6b, 6d and $6 \mathrm{f}$ are found to have high activity at all concentrations over $\mathrm{g}$ positive staphylococcus aureus. Compound 6a exhibited considerable activity over g negative bacterial strain E.Coli at all concentrations. Compounds $\mathbf{6 c}$ and $\mathbf{6 h}$ have responded with activity over g negative $P$. aeruginosa at 300 and $500 \mu$ g concentrations compared to the standard drug. The antifungal activity studies revealed that compound $\mathbf{6} \mathbf{d}$ has potential activity while compounds $\mathbf{6 a}$ and $\mathbf{6 e}$ have moderate activities and compound $\mathbf{6 h}$ showed weak activity against the fungi C.albicans. When compared to the standard (BHT).
The in vitro antibacterial studies of the oxadiazoles $\mathbf{7 a - h}$ (Table 2) revealed that compound $7 \mathbf{e}$ is moderate in activity while the remaining compounds did not announce any activity. The antifungal activity of the compounds 7a-h revealed that compound $7 \mathbf{e}$ exhibited good activity over the fungal species C.albicans at $500 \mu \mathrm{g}$ concentration while all the other remaining compounds are inert in activity. Based on the analytical results, it is evident that compound 6d (2-bromo substituted pyrazole hydrazone derivative) is a potential agent with significant antimicrobial activity.

\section{Anti-oxidant activity}

The antioxidant activity of the hydrazone and oxadiazole derivatives was evaluated using DPPH radical 
Table 3: Antioxidant activity of compounds 6a-h by DPPH method.

\begin{tabular}{|c|c|c|c|c|c|c|}
\multicolumn{7}{|c|}{ Table 3: Antioxidant activity of compounds 6a-h by DPPH method. } \\
\hline \multirow{2}{*}{ Compound } & $\mathbf{2 5} \boldsymbol{\mu M}$ & $\mathbf{5 0} \boldsymbol{\mu M}$ & $\mathbf{7 5} \boldsymbol{\mu M}$ & $\mathbf{1 0 0} \boldsymbol{\mu M}$ & $\mathbf{1 2 5} \boldsymbol{\mu M}$ & $\mathbf{2 5 0} \boldsymbol{\mu M}$ \\
\cline { 2 - 7 } & $11.9 \pm 1.53$ & $25.7 \pm 1.23$ & $36.1 \pm 1.32$ & $38.5 \pm 1.42$ & $39.4 \pm 1.18$ & $69.7 \pm 1.12$ \\
\hline $6 \mathrm{a}$ & $15.4 \pm 1.30$ & $27.7 \pm 1.24$ & $38.6 \pm 1.23$ & $39.3 \pm 1.36$ & $39.5 \pm 1.32$ & $69.8 \pm 1.43$ \\
\hline $6 \mathrm{~b}$ & - & - & - & - & - & - \\
\hline $6 \mathrm{c}$ & - & - & - & - & $37.34 \pm 1.54$ & $68.26 \pm 1.32$ \\
\hline $6 \mathrm{~d}$ & $12.2 \pm 1.03$ & $22.9 \pm 1.22$ & $34.3 \pm 1.47$ & $35.2 \pm 1.39$ & $35.5 \pm 1.35$ & $66.9 \pm 1.54$ \\
\hline $6 \mathrm{e}$ & $13.9 \pm 1.10$ & $25.8 \pm 1.15$ & $36.4 \pm 1.30$ & $36.9 \pm 1.43$ & $37.4 \pm 1.34$ & $68.3 \pm 1.36$ \\
\hline $6 \mathrm{f}$ & - & - & - & - & - & - \\
\hline $6 \mathrm{~g}$ & - & - & - & - & & - \\
\hline $6 \mathrm{~h}$ & $18.40 \pm 0.55$ & $28.28 \pm 1.20$ & $42.32 \pm 1.24$ & $45.35 \pm 1.32$ & $47.28 \pm 1.44$ & $76.38 \pm 1.32$ \\
\hline Control (BHT) & & & & & & - \\
\hline
\end{tabular}

\begin{tabular}{|c|c|c|c|c|c|c|}
\hline \multirow[b]{2}{*}{ Compound } & \multicolumn{6}{|c|}{ Scavenging activity $\left(\mathrm{IC}_{50}, \mu \mathrm{M}\right)$} \\
\hline & $25 \mu M$ & $50 \mu \mathrm{M}$ & $75 \mu \mathrm{M}$ & $100 \mu \mathrm{M}$ & $125 \mu \mathrm{M}$ & $250 \mu \mathrm{M}$ \\
\hline $7 a$ & - & - & - & - & - & - \\
\hline $7 \mathrm{~b}$ & - & - & - & - & - & - \\
\hline $7 c$ & - & - & - & - & - & - \\
\hline $7 d$ & - & - & - & - & $35.5 \pm 1.25$ & $66.9 \pm 1.48$ \\
\hline $7 e$ & $13.9 \pm 1.45$ & $25.4 \pm 1.34$ & $36.5 \pm 1.25$ & $38.1 \pm 1.37$ & $39.2 \pm 1.28$ & $69.9 \pm 1.32$ \\
\hline $7 f$ & $15.4 \pm 1.12$ & $25.8 \pm 1.32$ & $36.6 \pm 1.44$ & $38.8 \pm 1.33$ & $39.7 \pm 1.47$ & $69.8 \pm 1.29$ \\
\hline $7 \mathrm{~g}$ & - & - & - & - & - & - \\
\hline $7 \mathrm{~h}$ & - & - & - & - & - & - \\
\hline Control (BHT) & $18.40 \pm 0.55$ & $28.28 \pm 1.20$ & $42.32 \pm 1.24$ & $45.35 \pm 1.32$ & $47.28 \pm 1.44$ & $76.38 \pm 1.32$ \\
\hline
\end{tabular}

scavenging method using 2, 6-bis (1, 1-dimethylethyl)4-methylphenol (BHT) as standard.

The DPPH radical scavenging activity results of the hydrazone derivatives (6a-h) at different concentrations are depicted in Table 3 . The activity studies revealed that the compounds $\mathbf{6 a}, \mathbf{6 b}, \mathbf{6 e}$ and $\mathbf{6} \mathbf{f}$ exhibited strong antioxidant activities in all concentrations when compared to the standard BHT. Among the active compounds, compounds 6a (the phenyl substituted derivative) and compound $6 \mathrm{e}$ (the 2,5-dichloro substituted derivative) showed excellent activities, compound $\mathbf{6} \mathbf{a}$ being the most potent compound. Next to $\mathbf{6} \mathbf{a}$ and $\mathbf{6 e}$, compounds $\mathbf{6} \mathbf{b}$ and $\mathbf{6 f}$ exhibited promising activities while compound 6d showed good activity at $125 \mu \mathbf{M}$ and $250 \mu \mathbf{M}$ concentrations. The order of radical scavenging activities of these compounds is in the order $\mathbf{6} a>6 \mathbf{6 e}>\mathbf{6} f>\mathbf{6 b}>\mathbf{6 d}$. All the remaining compounds announced non antioxidant activity.

The DPPH radical scavenging activity results of the oxadiazole derivatives (7a-h) at different concentrations are depicted in Table 4. From the Table, it is evident that the compounds $7 \mathbf{e}(2,5$-dichloro) and $7 \mathbf{f}$ (3,5-dichloro) exhibited excellent anti oxidant activities, compound 7e being the most potent compound when compared to the standard BHT. Compound $\mathbf{7 d}$ showed good activity at $125 \mu \mathrm{M}$ and $250 \mu \mathrm{M}$ concentrations while the remaining compounds announced non- antioxidant activity.

\section{CONCLUSION}

Screening of compounds $\mathbf{6 a - h}$ and $\mathbf{7 a - h}$ invitro for their anti-microbial activity against Streptomycin as control drug indicated that the compound $\mathbf{6 d}$ (2-bromo substituted pyrazole hydrazone derivative) is antimicrobial potent. The antioxidant evaluation of the same series of compounds using DPPH radical scavenging method revealed that compounds $\mathbf{6 a}, \mathbf{6 e}, \mathbf{7 e}$ and $\mathbf{7 f}$ announced strong activities when compared to the control BHT.

\section{ACKNOWLEDGEMENT}

The authors sincerely express their gratitude Dr. P. Yadagiri Reddy, CEO, Escientia Bio Pharma Pvt. Ltd, Hyderabad for his encouragement in completing the present research work. The authors extend their sincere thanks 
to Dr. D. Jayasimharayalu, Director, Global institute of Bio Technology, Himayathnagar, Hyderabad for his cooperation in carrying out the anti bacterial and anti oxidant studies.

\section{CONFLICT OF INTEREST}

The authors declare that there is no conflict of interest.

\section{ABBREVIATIONS USED}

TLC: Thin layer chromatography; FT-IR: Fourier transform infrared spectroscopy; NMR: Nuclear magnetic resonance spectroscopy; DMSO-d $\mathrm{d}_{6}$ Deuterated dimethyl sulphoxide; HRMS: High resolution mass spectrometry; M.P: Melting Point; DPPH: 2, 2-diphenyl-1-picrylhydrazyl; BHT: 2, 6-bis (1, 1-dimethylethyl)4-methylphenol; BOD: Bio Chemical Oxygen Demand.

\section{REFERENCES}

1. Shi W, Qian X, Song G, Zhang R, Li R. Syntheses and insecticidal activities of novel 2-fluorophenyl-5-aryl/cyclopropyl-1, 3, 4-oxadiazoles. J Fluorine Chem. 2000; 106(2):173-9. https://doi.org/10.1016/S0022-1139(00)00323-7.

2. Diana GD, Volkots DL, Nitz TJ, Bailey TR, Long MA, Vescio N, Aldous S, et al,. Oxadiazoles as ester bioisosteric replacements in compounds related to disoxaril. Antirhinovirus activity. J Med Chem.1994; 37(15):2421-36. https://doi.org/10.1021/jm00041a022. PMID: 8057290.

3. Behalo MS. An efficient one-pot catalyzed synthesis of 2, 5-disubstituted-1, 3, 4-oxadiazoles and evaluation of their antimicrobial activities. RSC Adv. 2016; 6(105):03132-6. https://doi.org/10.1039/C6RA22663A.

4. Mihailovic N, Markovic V, Matic IZ, Stanisavljevic NS, Jovanovic ZS, Trifunovic S. et al,. Synthesis and antioxidant activity of 1, 3, 4-oxadiazoles and their diacylhydrazine precursors derived from phenolic acids. RSC Adv. 2017; 7(14): 8550-60. https://doi.org/10.1039/C6RA28787E.

5. Gamal El-Din MM, El-Gamal MI, Abdel-Maksoud MS, Yoo KH, Oh CH. Synthesis and in vitro antiproliferative activity of new 1, 3, 4-oxadiazole derivatives possessing sulfonamide moiety. Eur J Med Chem. 2015; 90:45-52. https://doi.org/10.1016/j.ejmech.2014.11.011. PMID: 25461310.

6. Rajak H, Singour P, Kharya MD, Mishra P. A novel series of 2, 5-disubstituted 1, 3, 4-oxadiazoles: Synthesis and SAR study for their anticonvulsant activity. Chem Biol Drug Des. 2011; 77(2):152-8. https://doi.org/10.1111/j.17470285.2010.01066.x. PMID: 21266018

7. Dhumal ST, Deshmukh AR, Bhosle MR, Khedkar VM, Nawale LU, Sarkar D, et al,. Synthesis and antitubercular activity of new 1, 3, 4-oxadiazoles bearing pyridyl and thiazolyl scaffolds. Bioorg Med Chem Lett. 2016; 26(15):3646-51. https://doi.org/10.1016/j.bmcl.2016.05.093. PMID: 27301367.

8. Wu W, Chen Q, Tai A, Jiang G, Ouyang G. Synthesis and antiviral activity of 2-substituted methylthio-5-(4-amino-2-methylpyrimidin-5-yl)-1, 3, 4-oxadiazole derivatives. Bioorg Med Chem. Lett. 2015; 25(10): 2243-6. https://doi. org/10.1016/j.bmcl.2015.02.069. PMID: 25900217.

9. Zareef M, lqbal R, De Dominguez NG, Rodrigues J, Zaidi JH, Arfan M, et al,. Synthesis and antimalarial activity of novel chiral and achiral benzenesulfonamides bearing 1,3, 4-oxadiazole moieties. JEnzyme InhibMed Chem. 2007; 22(3):301-8. http://dx.doi.org/10.1080/14756360601114569. PMID: 17674812.

10. Biju CR, llango K, Manju P, Rekha K. Design and microwave-assisted synthesis of 1, 3, 4-oxadiazole derivatives for analgesic and anti-inflammatory activity. J Young Pharm. 2012; 4(1):33-7. https://doi.org/ 10.4103/09751483.93576. PMCID: PMC3326780

11. Plant HL, Regis RR, Moore RC. Substituted oxadiazole-Phosphate pesticide. 1985; Eur. Pat. Appl. EP 156,638 A2.
12. Matthews IR, Bacon DP. Thia-and oxadiazole derivatives and their use as fungicides or insecticides. 1995; PCT Int. Appl. WO 9505368 A1.

13. Kraatz U, Gallenkamp B, Marhold A, Wolfrum P,Andersch W, Erdelen C, et al,. Heterocyclic Fluoroalkenyl Thioethers. 2002; WO 2002006256 A1.

14. W. Wiedemann, Chem. Z. 106 (1982) 313.

15. Gomes D, Borges CP, Pinto JC. Study of the synthesis of poly (4, 4'-diphenylether-1, 3, 4-oxadiazole) in solutions of poly (phosphoric acid). Polymer. 2001; 42(3):851-65. https://doi.org/10.1016/S0032-3861(00)00458-4.

16. Meier M, Buchwald E, Karg S, Posch P, Greczmiel M, Strohriegl P, et al,. Heterolayer light-emitting diodes based on new oxadiazole polymers. Synth Met. 1996; 76 :( 1-3)95-9. https://doi.org/10.1016/0379-6779(95)03426-K

17. Malladi S, Isloor AM, Peethambar SK, Fun HK. Synthesis and biological evaluation of newer analogues of 2, 5-disubstituted 1, 3, 4-oxadiazole containing pyrazole moiety as antimicrobial agents. Arab J Chem. 2014; 7(6): 1185-91. https://doi.org/10.1016/j.arabjc.2013.12.020.

18. Renuka N, Vivek HK, Pavithra G, Ajay Kumar K. Synthesis of coumarin appended pyrazolyl-1, 3, 4-oxadiazoles and pyrazolyl-1, 3, 4-thiadiazoles: Evaluation of their in vitro antimicrobial and anti oxidant activities and molecular docking studies. Russ J Bioorg Chem. 2017; 43(2): 197-210. https://doi.org/ 10.1134/S106816201702011X.

19. Kumar M, Kumar V, Beniwal V. Synthesis of some pyrazolylaldehyde $\mathrm{N}$-isonicotinoyl hydrazones and 2, 5-disubstituted 1, 3, 4-oxadiazoles as DNA photocleaving agents. Med Chem Res. 2015; 24(7): 2862-70. https:// doi.org/10.1007/s00044-015-1340-x.

20. Puthiyapurayil P, Poojary B, Chikkanna C, Buridipad SK. Design, synthesis and biological evaluation of a novel series of 1,3,4-oxadiazole bearing $\mathrm{N}$-methyl-4-(trifluoromethyl) phenyl pyrazole moiety as cytotoxic agents. Eur J Med Chem. 2012; 53:203-10. https://doi.org/10.1016/j.ejmech.2012.03.056. PMID: 22542958.

21. Bansal S, Bala M, Suthar SK, Choudhary S, Bhattacharya S, Bhardwaj $\mathrm{V}$, et al,. Design and synthesis of novel 2-phenyl-5-(1, 3-diphenyl-1Hpyrazol-4-yl)-1, 3, 4-oxadiazoles as selective COX-2 inhibitors with potent anti-inflammatory activity. Eur J Med Chem. 2014; 80:167-74. https://doi. org/10.1016/j.ejmech.2014.04.045. PMID: 24780593

22. Farghaly AR, El-Kashef $H$. Synthesis of some new azoles with antiviral potential. ARKIVOC. 2006; 11:76-90. https://doi.org/ 10.3998/ ark.5550190.0007.b07.

23. Rollas S, Kucukguzel SG. Biological Activities of hydrazone derivatives. Molecules. 2007; 12(8):1910-39. https://doi.org/10.3390/12081910. PMID: 17960096

24. Ajani OO, Obafemi CA, Nwinyi OC, Akinpelu DA. Microwave assisted synthesis and antimicrobial activity of 2-quinoxalinone-3-hydrazone derivatives. Bioorg Med Chem. 2010; 18(1):214-21. https://doi.org/10.1016/j. bmc.2009.10.064. PMID: 19948407.

25. El-Bayoumi MA, El-Aasser M, Abdel-Halim F. Electronic spectra and structures of Schiff's bases. I. Benzanils. J Am Chem Soc. 1971; 93(3):586-90. https://doi.org/10.1021/ja00732a004.

26. Jedlovska E, Lesko J. A simple one-pot procedure for the synthesis of 1,3 , 4-Oxadiazoles. Synth Commun. 1994; 24(13): 1879-85. http://dx.doi. org/10.1080/00397919408010196.

27. Popiolek L. Hydrazide-hydrazones as potential antimicrobial agents: overview of the literature since 2010. Med Chem Res. 2017; 26(2):287-01. DOI 10.1007/s00044-016-1756-y. PMCID: PMC5250660.

28. Belkheiri N, Bouguerne B, Bedos-Belval F, Duran H, Bernis C, Salvayre R, et al,. Synthesis and antioxidant activity evaluation of a syringic hydrazones family. Eur J Med Chem. 2010; 45(7):3019-26. https://doi.org/10.1016/j. ejmech.2010.03.031. PMID: 20403645.

29. Angelova VT, Valcheva V, Pencheva T, Voynikov Y, Vassilev N, Mihaylova R, et al,. Synthesis, antimycobacterial activity and docking study of 2-aroyl-[1] benzopyrano[4,3-c]pyrazol-4(1H)-one derivatives and related hydrazidehydrazones. Bioorg Med Chem Lett. 2017; 27(13):2996-3002. https://doi. org/10.1016/j.bmcl.2017.05.011. PMID: 28512022.

30. Demirbas N, Karaoglu SA, Demirbas A, Sancak K. Synthesis and antimicrobial activities of some new 1-(5-phenylamino-[1,3,4]thiadiazol-2yl)methyl-5-oxo-[1,2,4]triazole and 1-(4-phenyl-5-thioxo-[1,2,4]triazol-3-yl)methyl-5-oxo- [1,2,4] triazole derivatives. Eur J Med Chem. 2004; 39(9):793804. https://doi.org/10.1016/j.ejmech.2004.06.007. PMID: 15337292 
31. Li ZH, Yang DX, Geng PF , Zhang J, Wei HM, Hu B, et al,. Design, synthesis and biological evaluation of $[1,2,3]$ triazolo[4,5-d]pyrimidine derivatives possessing a hydrazone moiety as antiproliferative agents. Eur J Med Chem. 2016; 124:967-80. https://doi.org/10.1016/j.ejmech.2016.10.022. PMID: 27771599

32. Kumar N, Chauhan LS, Sharma CS, Dashora N, Bera R. Synthesis, analgesic and anti-inflammatory activities of chalconyl-incorporated hydrazone derivatives of mefenamic acid. Med Chem Res. 2015; 24(6):2580-90. https:// doi.org/ 10.1007/s00044-015-1318-8.

33. Liu Y, Lu B, Lu J, Xin C, Li J, Mu J, et al,. Synthesis and antibacterial activities of $\mathrm{N}$-[(1-aryl-3-phenyl-pyrazol-4-yl) methylene]-2-(halo-o-hydroxyphenyl) hydrazide derivatives. Chem Res Chin Univ. 2013; 29(3):449-53. https://doi. org/ 10.1007/s40242-013-2491-2.

34. Yang XD. Synthesis and biological activity of hydrazone derivatives containing pyrazole. J Chem Res. 2008; 9:489-91. https://doi. org/10.3184/030823408X340799.

35. Xing M, Zhao TT, Ren YJ, Peng NN, Yang XH, Li X, et al,. Synthesis, biological evaluation, and molecular docking studies of pyrazolylacylhydrazone derivatives as novel anticancer agents. Med Chem Res. 2014; 23(7):3274-86. https://doi.org/ 10.1007/s00044-014-0909-0.

36. Nasrullah M, Khan MA, Khan MN, Humphrey MG, Nasim FH, Chaudhry F, et al,. Diaryl pyrazole-4-carbaldehyde benzoylhydrazones metal complexes: Synthesis and their antibacterial and antioxidant screening. Asian $\mathrm{J}$ Chem. 2013; 25(1):419-23. https://doi.org/10.14233/ajchem.2013.13131.

37. El-Sayed MA, Abdel-Aziz NI, Abdel-Aziz AA, El-Azab AS, Asiri YA, ElTahir $\mathrm{KEH}$. Design, synthesis, and biological evaluation of substituted hydrazone and pyrazole derivatives as selective COX-2 inhibitors: Molecular docking study. Bioorg Med Chem. 2011; 19(11):3416-24. https://doi.org/10.1016/j. bmc.2011.04.027. PMID: 21570309.

38. Abadi AH, Eissa AAH, Hassan GS. Synthesis of novel 1, 3, 4-trisubstituted pyrazole derivatives and their evaluation as antitumor and antiangiogenic agents. Chem Pharm Bull. 2003; 51(7):838-44. http://doi.org/10.1248/ cpb.51.838. PMID: 12843591.

39. Fraga AGM, Rodrigues CR, Miranda ALP, Barreiro EJ, Fraga CAM. Synthesis and pharmacological evaluation of novel heterocyclic acylhydrazone derivatives, designed as PAF antagonists. Eur J Pharm Sci. 2000; 11(4): 285-90. https://doi.org/10.1016/S0928-0987(00)00102-0. PMID: 11033071.

40. Bandgar BP, Totre JV, Gawande SS, Khobragade CN, Warangkar SC, Kadam PD. Synthesis of novel 3, 5-diaryl pyrazole derivatives using combinatorial chemistry as inhibitors of tyrosinase as well as potent anticancer, antiinflammatory agents. Bioorg Med Chem. 2010; 18(16):6149-55. https://doi. org/10.1016/j.bmc.2010.06.046. PMID: 20638287

41. Ragavan RV, Vijayakumar V, Kumari NS. Synthesis and antimicrobial activities of novel 1, 5-diaryl pyrazoles. Eur J Med Chem. 2010; 45(3):1173-80. https://doi.org/10.1016/j.ejmech.2009.12.042. PMID: 20053480.

42. Bellam M, Gundluru M, Sarva S, Chadive S, Netala VR, Tartte V, et al,. Synthesis and antioxidant activity of some new $\mathrm{N}$-alkylated pyrazolecontaining benzimidazoles. Chem Heterocycl Comp. 2017; 53(2):173-78. https://doi.org/ 10.1007/s10593-017-2036-6.

43. Alegaon SG, Alagawadi KR, Garg MK, Dushyant K,Vinod D. 1, 3, 4-Trisubstituted pyrazole analogues as promising anti-inflammatory agents. Bioorg Chem. 2014; 54: 51-9. https://doi.org/10.1016/j.bioorg.2014.04.001. PMID: 24793214.

44. Pathak V, Maurya HK, Sharma S, Srivastava KK, Gupta A. Synthesis and biological evaluation of substituted 4, 6-diarylpyrimidines and 3, 5-diphenyl- 4,5-dihydro- $1 \mathrm{H}$-pyrazoles as anti-tubercular agents. Bioorg Med Chem Lett. 2014; 24(13):2892-6. https://doi.org/10.1016/j.bmcl.2014.04.094. PMID: 24835631.

45. Ali AR, El-Bendary ER, Ghaly MA, Shehata IA. Synthesis, in vitro anticancer evaluation and in silico studies of novel imidazo[2,1-b]thiazole derivatives bearing pyrazole moieties. Eur J Med Chem. 2014; 75:492-500. https://doi. org/10.1016/j.ejmech.2013.12.010. PMID: 24576591.

46. El-Sabbagh OI, Baraka MM, Ibrahim SM, PannecouqueC, Andrei G, Snoeck R, et al,. Synthesis and antiviral activity of new pyrazole and thiazole derivatives. Eur J Med Chem. 2009; 44(9):3746-53. https://doi.org/10.1016/j. ejmech.2009.03.038. PMID: 19419804.

47. Abdel-Aziz M, Abuo-Rahma Gel-D, Hassan AA. Synthesis of novel pyrazole derivatives and evaluation of their antidepressant and anticonvulsant activities. Eur J Med Chem. 2009; 44(9):3480-7. https://doi.org/10.1016/j. ejmech.2009.01.032. PMID: 19268406.

48. Genin MJ, Biles C, Keiser BJ, Poppe SM, Swaney SM, Tarpley WG, et al,. Novel 1, 5-Diphenylpyrazole nonnucleoside HIV-1 reverse transcriptase inhibitors with enhanced activity versus the delavirdine-resistant P236L mutant: Lead identification and SAR of 3- and 4-substituted derivatives. J Med Chem. 2000; 43(5):1034-40. https://doi.org/ 10.1021/jm990383f. PMID: 10715167.

49. Penning TD, Talley JJ, Bertenshaw SR, Carter JS, Collins PW, Docter S, et al. Synthesis and biological evaluation of the 1, 5-diarylpyrazole class of cyclooxygenase-2 Inhibitors: Identification of 4-[5-(4-Methylphenyl)-3(trifluoromethyl)-1H-pyrazol-1-yl]benzenesulfonamide (SC-58635, Celecoxib). J Med Chem. 1997; 40(9):1347-1365. https://doi.org/10.1021/ jm960803q. PMID: 9135032.

50. Hote BS, Lokhande PD. Novel and efficient synthesis of 4-indazolyl-1, 3, 4-trisubstituted pyrazole derivatives. Synth Commun. 2014; 44(10):1492500. http://dx.doi.org/10.1080/00397911.2013.862724.

51. Kumar BC, Reddy KRV, Khan F. Synthesis, characterization and antimicrobial activity of some new N1-substituted pyrazol-4-carbaldehyde bearing 2, 4-dichloro phenyl moiety. J Pharm Sci. 2013; 2(5):55-9. http://dx.doi.org/ 10.7897/2277-4572.02578.

52. Han S, Zhang FF, Xie X, Chen JZ. Design, synthesis, biological evaluation, and comparative docking study of 1, 2, 4-triazolones as CB1 receptor selective antagonists. Eur J Med Chem. 2014; 74: 73-84. http://dx.doi. org/10.1016/j.ejmech.2013.12.018. PMID: 24445310.

53. Yu W, Huang G, Zhang Y, Liu H, Dong L, Yu X, et al,. I $\mathrm{I}_{2}$-mediated oxidative C-O bond formation for the synthesis of 1, 3, 4-oxadiazoles from aldehydes and hydrazides. J Org Chem. 2013; 78(20):10337-43. http://dx.doi. org/10.1021/jo401751h. PMID: 24059837.

54. Rahman AU, Choudhary MI, Thomsen WJ. Bioassay techniques for drug development. Harwood academic publishers. Netherlands. Harwood academic publisher; 2001; 22:16.

55. Yen GC, Chen HY. Antioxidant activity of various tea extracts in relation to their antimutagenicity. J Agric Food Chem. 1995; 43(1):27-32. http://dx.doi. org/ 10.1021/jf00049a007.

56. Lopes AB, Miguez E, Kümmerle AE, Rumjanek VM, Fraga CA, Barreiro EJ. Characterization of amide bond conformers for a novel heterocyclic template of N-acylhydrazone derivatives. Molecules 2013; 18(10):11683-704; doi: 10.3390/molecules181011683. PMID: 24071978. 
PICTORIAL ABSTRACT

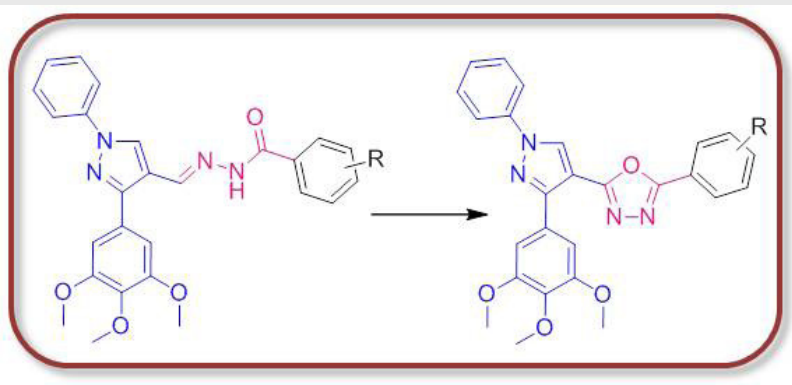

\section{About Authors}

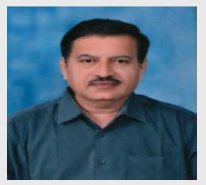

Dr. P.V.S. Machiraju is presently working as a professor of chemistry and Dean-R and D, Pragathi Engineering College (Autonomous) Surampalem, near Kakinada City, A.P. Research Interests: Include Organic, Analytical, Environmental and Bio Chemistry. Also in interdisciplinary with Hydrology, Microbiology, Nano Chemistry and Technology. He has 38 international and 6 national publications at his career.

\section{SUMMARY}

- A novel 1, 3, 4-oxadiazole substituted pyrazole scaffolds 7a-h were synthesized by the iodine catalyzed oxidative cyclization of different hydrazone derivatives $\mathbf{6 a}-\mathbf{h}$ in good to excellent yields. The hydrazone derivatives $\mathbf{6 a}-\mathbf{h}$ were obtained by heating the corresponding aldehyde intermediate 4 in ethanol with various substituted aryl hydrazides. All the final compounds were characterized by IR, NMR and HRMS spectral analysis. The invitro antimicrobial activity of the synthesized compounds 6a-h and 7a-h were screened over two g negative bacterial strains E.Coli, $P$. aeruginosa and two $\mathrm{g}$ positive bacterial strains $S$. aureus, $B$. cereus with three different concentrations of 200,300 and 500 $\mu \mathrm{g} / \mathrm{mL}$ using Streptomycin as standard drug. The study results revealed that the compounds $\mathbf{6 a}, \mathbf{6 b}$, $\mathbf{6 d}$ and $\mathbf{6 f}$ are with moderate to good antimicrobial activity. The compounds $\mathbf{6 a}, \mathbf{6 d}$ and $\mathbf{6 e}$ are found to have moderate to good antifungal activity. Finally the research study results revealed that the compound 6d (2-bromo substituted pyrazole hydrazone derivative) as a potential antimicrobial agent. Furthermore, the antioxidant activity studies revealed that the compounds $6 \mathrm{a}, \mathbf{6 e}$ and $7 \mathrm{e}$, 7f exhibited potent anti oxidant activities when compared to the control drug BHT.

Cite this article: Ch.Krishna Prasad, Machiraju PVS. Synthesis and Characterization of 2-phenyl-5-(1-phenyl-3-(3, 4, 5-trimethoxyphenyl)-1H-pyrazol-4-yl) - 1, 3, 4-oxadiazole Scaffolds for Assessing Their Medicinal Potentials. Indian $\mathrm{J}$ of Pharmaceutical Education and Research. 2018;52(1):135-45. 